\title{
17 \\ A Multimedia Database System VHM and Its Application
}

\author{
Shoichiro Ishigaki \\ Multimedia Service Promotion Headquarters NTT \\ Hibiya Kokusai Bldg. 2-2-3 Uchisaiwai-chou Chiyoda-ku 100 Tokyo \\ Japan, Tel:+8133509 5405,Fax:+813 5512 7497, Fax: 01223-439585, \\ email: ishigaki@mod.hqs.ntt.jp \\ Kazuhiko Kushima and Tetsuo Sakata \\ NTT Information and Communication Systems Laboratories \\ 1-2356 Take Yokosuka-shi Kanagawa 238-03 Japan, Tel:+81 46859 \\ 2751,Fax:+8146855 1152, e-mail: \{kushima, sakata\}@dq.isl.ntt.jp
}

\begin{abstract}
Video Hyper Media (VHM) is an environment for creating and executing interactive multimedia applications. The database of the VHM comprises multimedia contents such as still pictures, moving pictures, and sounds and scenarios that describe the behavior and dynamic relationships of compound objects (combinations of the multimedia contents). With the interactive edit function of the VHM, applications such as the on-screen subject search and a video walk-through application can be developed without any programming. As an example of an application created with the VHM, we developed a railway line information system and confirmed the effectiveness of the VHM.
\end{abstract}

Multimedia, database, interaction, scenario, model

\section{INTRODUCTION}

Several problems exist in creating interactive multimedia applications, that is, it is costly and time consuming to create the contents and applications. The reasons why it is costly and time consuming can be summarized as follows:

1. Unlike non-interactive applications such as video, interaction with users (interactive behavior) must be defined in interactive multimedia applications. In many of the existing systems, however, this interactive behavior is defined through programming. In other words, user actions and system responses are all specified through programming.(Hodge 1993) 
2. Supporting functions in order to share or reuse those interactive behaviors are not provided. Therefore, everything must be created from scratch in developing applications.

The problem inherent in (1) is the method for describing applications. Developing a high-level description model and providing a programless creation system of the model have been found to be effective. We call this description the scenario. As for (2), it is necessary to develop a mechanism for sharing media data and parts of the scenarios (subscenarios) among applications. In this paper, a collection of scenarios and media data to be shared is called a multimedia database.

In this paper, we propose a Video Hyper Media (VHM) model to describe multimedia applications. In this model, the scenario is a hierarchical and directed graph with displayed scenes as nodes which are connected to selective links. (Sakata 1996)

We also propose a VHM database as a mechanism to store and reuse the VHM model. The VHM database manages the scenarios created using the VHM model and media data used in the scenarios. The VHM database enables sharing of the scenarios among several users and reuse of subscenarios.

Finally, we describe a railway line information system as an implementation example of the model and discuss its effectiveness.

\section{STRUCTURE AND DESCRIPTION OF SCENARIOS}

In interactive multimedia applications, it is necessary to define user actions and system responses.(Nielsen 1990) Such defined information is called a scenario. Various selection methods among system responses are required in scenarios depending on the user requests.

Simple selection Users can select and replay the desired media data and scenarios by clicking on an interesting subject from the menus shown on the screen.

Video walk-through Based on an actual video of a wide space such as a street, users can experience a virtual walk-through scenario such as turning at an intersection by selecting a desired direction and entering a store.

On-screen subject search Users can search for a subject on the screen, e.g., obtaining detailed information on a swimming fish by touching the fish itself rather than by specifying the name of the fish.

The above selection methods can be expressed in a uniform way by treating user's actions as events. Table 1 shows examples of the necessary events.

These events cause a transition from one picture to another. For example, in the video walk-through application, user actions at an intersection can be expressed as in Figure 1.

The definition of an interaction based on events can be expressed in a directed graph by using the node link model. In other words, the interaction can be defined by specifying the node to be replayed and associating the event to a link. Figure 2 shows the expression of the node link model for handling the events at an intersection. Users can continue on to the next picture as branching-off to the left, right, up, or down from the intersection occurs according to the contents of each link.

Actions of users and responses of the system can be expressed as a static data structure using the node link model. Thus, applications can easily be created by describing scenarios 


\begin{tabular}{lll}
\hline Type of event & Timing of event & Example \\
\hline Selection & Click on a button on the screen & $\begin{array}{l}\text { Selecting a menu, specifying a di- } \\
\text { rection in the video walk-through }\end{array}$ \\
\hline Specify subject & Click on a subject on the video & $\begin{array}{l}\text { Selecting a swimming fish in the } \\
\text { Aquarium }\end{array}$ \\
\hline
\end{tabular}

Table 1 Example of the necessary events

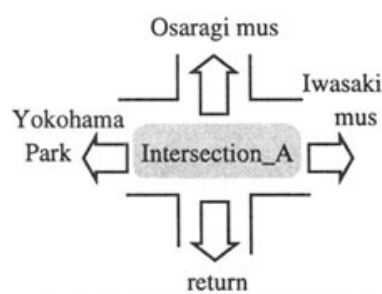

An intersection in the Real World

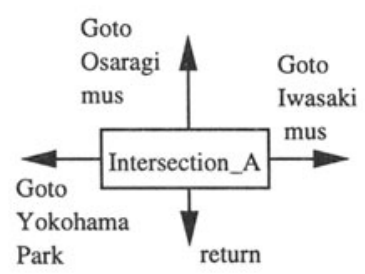

Events and actions at the intersection

Figure 1 User's action model at an intersection.
Scenario

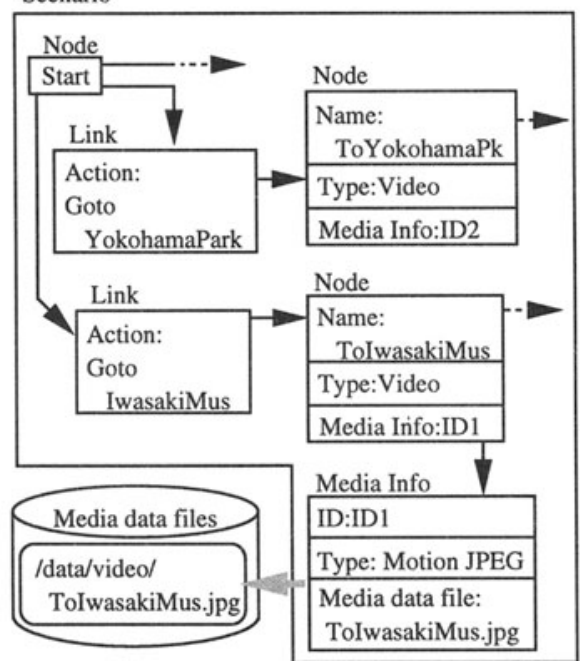

Figure 2 Node-link model for handling user's action.

in the creation support system and interpreting the defined scenarios in the execution system. The development time can be extremely reduced by modifying scenarios and verify its behavior interpretively.

\section{VHM CONFIGURATION}

The VHM comprises the VHM editor, MMDBMS, and an interactive player (Figure 3). 


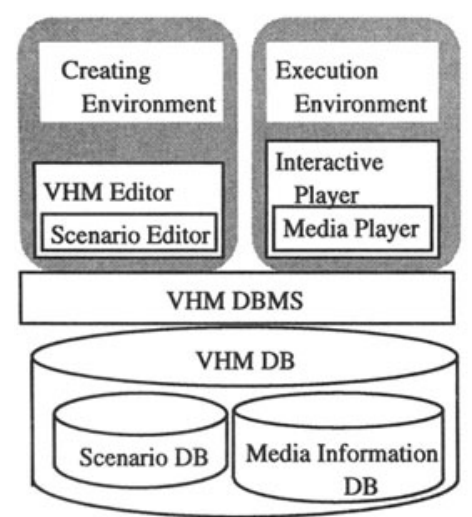

Figure 3 Structure of VHM System.

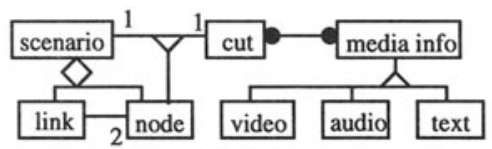

Schema of VHM DB

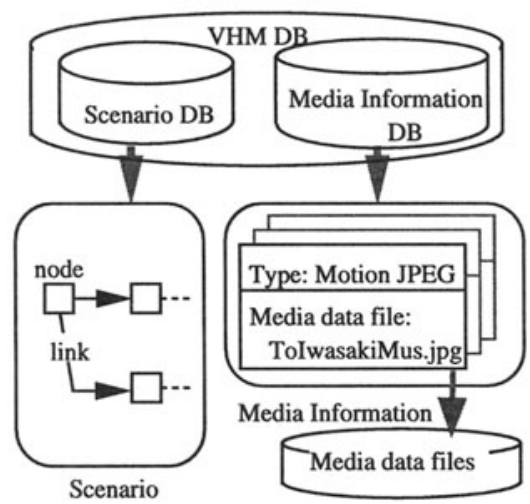

Figure 4 Structure of VHM DB.

VHM editor Creating multimedia applications is costly because scripts must be described to create scenarios. The VHM provides a programless creation environment based on a GUI so that a application developer who does not have knowledge of programming can easily create scenarios.

VHM database The VHM database manages the scenarios created by the VHM editor and provides the access functions for interactive player.

Interactive player The interactive player unfolds a scenario on the time axis and determines which media data are replayed in what order.

\section{DATABASE STRUCTURE}

The VHM database stores scenarios together with media information. Figure 4 shows the database structure.

\subsection{Database Schema}

The schema in the VHM incorporates structuring components such as nodes and links which are necessary for expressing scenarios. 
1. Each node expresses media information such as cuts and sounds from video pictures and still pictures. To achieve a hierarchical scenario structure, nodes are enabled to incorporate subordinate scenarios recursively.

2. Links define user actions and subsequent system responses. The links express one of the following:

Successive execution indicates a node to be executed after another node.

Parallel execution indicates a node to be executed in parallel with another node.

Selection indicates that one of the branch nodes is selected by an event from a user or the system.

On-screen subject search indicates that the subject on the screen itself has links to other nodes.

\subsection{Management through Database}

The VHM database enables sharing of scenarios and reuse of subscenarios among several users.

\section{Management of Scenarios}

In large-scale application development, scenarios must be created in a hierarchical structure. Scenarios must also be modularized from the viewpoint of sharing and reusing them. Therefore, VHM scenarios have the following characteristics:

- Hierarchical relationships among scenarios can be defined to facilitate application development. Thus, application developers can start with the top layer and gradually work on the details. Dispersed development, i.e., development done by a large number of people remotely located is also possible.

- VHM scenarios have special entrance and exit nodes to guarantee scenario modularity. A series of scenarios starting from the entrance node continuing to the exit node can be reused. Scenarios are expressed in a simple data structure using the object-oriented DBMS in order to facilitate sharing and reusing.

All nodes and links are expressed as objects and combined with object IDs. A series of scenarios can be quickly searched by tracing them from the entrance node through the combinations with object IDs. In nodes that indicate replaying media data, media information is referred to instead of the media data themselves so that changes in the media data can be flexibly handled.

\section{Managing Media Information}

Video information, for example, should have the attribute information including the video format information, the start and end time in the entire video, and other media data to be synchronized (e.g., sound). The VHM manages the media information storing such attributes in addition to the media data file names. This media information management increases the independence of the scenarios from the raw materials. 


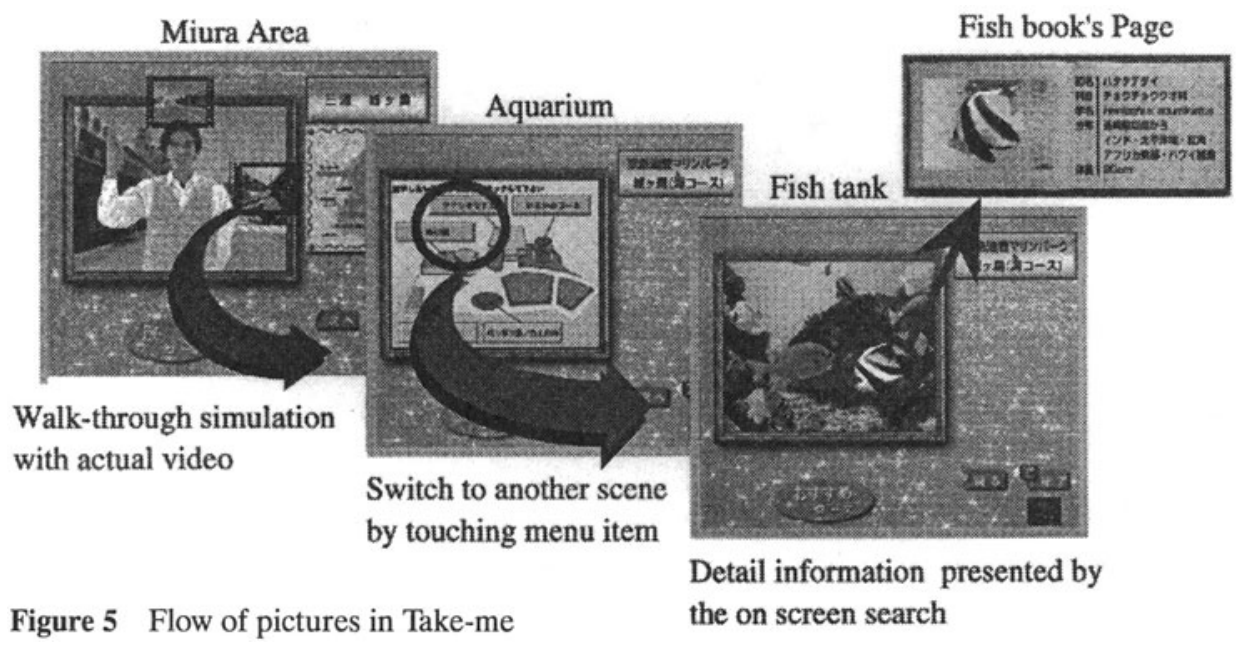

\section{INTERACTIVE PLAYER}

The interactive player not only unfolds the given scenario but also absorbs the differences in hardware dependent output devices (so called media players). It sends out the media data to the appropriate media player. Also, the interactive player has the following characteristics to achieve interactive operation.

Uninterruptible Replay of Video In the video walk-through application, it is necessary to replay the video of a specified direction immediately after a user selects the desired direction at an intersection. To do this, the interactive player has several media players standing by for all possible directions to provide uninterruptible replay.

Multi-stream Replay The interactive player is capable of replaying more than one video at a time by running several media players in parallel.

\section{RAILWAY LINE INFORMATION SYSTEM - TAKE-ME -}

This chapter describes the railway line information system called Take-me as an implementation example of the VHM and discusses its effectiveness. Take-me is a railway line information system using a wide screen. Users select the desired menu from the guide provided on a terminal by touching the screen in order to obtain tourist information on a railway line. The same picture is shown on a multiscreen with nine displays so that several people can see it at the same time. Take-me is a commercial system created using VHM and is currently operating at several stations on a private railway line. Figure 5 shows shows the flow of the pictures in Take-me. 


\subsection{Interactive Functions of VHM}

Simultaneous Display of Multimedia Information The initial menu screen shows menus comprising several videos at the same time in a thumb-nail sketch to aid users in making a selection. In addition, in the railway line information system, the combination of background music and a description with text and pictures is also used.

User Selection Take-me is installed at a station and used by an unspecified number of the general public. Thus, all operations are done simply by touching a button or subject on the screen.

Video Walk-through-Type Railway Line Information System Users can walk through major attractions on a railway line as they watch the actual video. In the walk-through process with combinations of actual pictures, users can stop at each intersection and indicate which direction to go. The system also provides a function to show an abbreviated version for users with limited time.

On-screen Subject Search at an Aquarium In the aquarium of Take-me, users can perform an on-screen subject search on a fish swimming in a tank. A description of the fish appears when a user touches the fish swimming on the screen.

\subsection{Discussion}

We succeeded in creating scenarios for Take-me in a uniform way by using the VHM model.

1. Figure 6 shows the scenario for the railway line tourist information system using the video walk-through application. User selections in the railway line information system are mapped on a link on the scenario. Selections and on-screen subject search by an end user are mapped in the same way. By handling the events in a uniform way, end users can give any instruction by simply touching the desired object on the screen. A touch on the screen can be a turn at an intersection or an operation to obtain information on a particular fish.

2. By using the database management, engineers who develop scenarios are able to build a hierarchical structure and reuse it. Therefore, applications were developed within a short period of time. For example, it is easy to create the video walk-through scenarios by sharing the structure description at intersections and by switching the media information.

\section{CONCLUSION}

We proposed a framework called the Video Hyper Media and developed a data model to achieve it. The VHM structures and presents the multimedia information to enable user interaction. We also developed a railway line information system as an application on the VHM. It showed that the programless creation environment and the sharing of scenarios in a database facilitates the application development. 


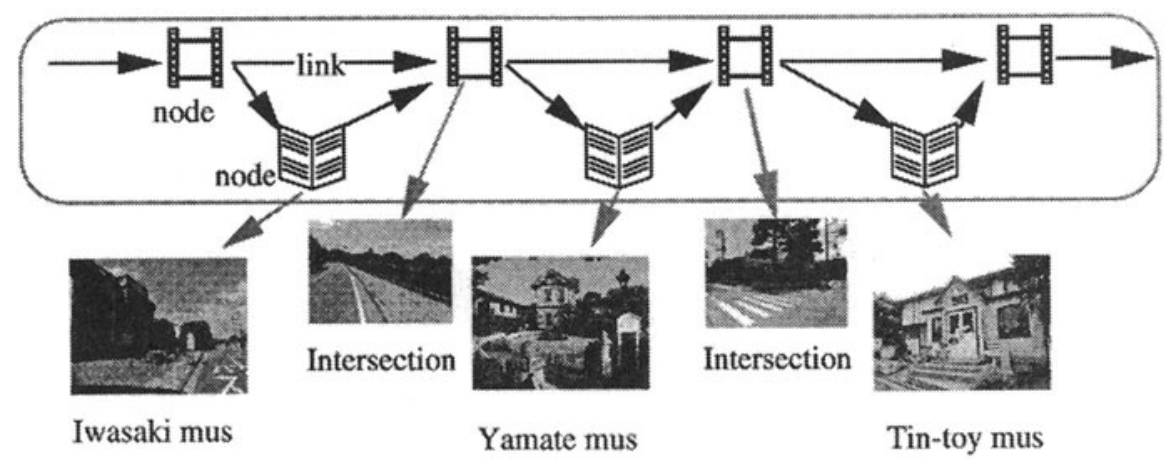

Figure 6 Scenario for Walk-through around Yokohama park

\section{REFERENCES}

Hodges, M.E. and Sasnett, R.M. (1993) Multimedia Computing: Case studies from MIT Project Athena, Addison Wesley, New York.

Nielsen, J. (1995) Multimedia and Hypertext : The internet and beyond, Ch.13, Academic Press Inc.,New York.

Sakata, T., Kojima, A. and Satoh, T. (1996) Hyper Video: Video Based Hypermedia for Walk-through Applications, Multimedia Japan 96, pp 148-154.

\section{BIOGRAPHY}

Shoichiro Ishigaki is a Senior Manager, Multimedia Service Department Multimedia Service Promotion Headquarters, NTT. He engaged in research on multimedia database systems and their applications. He received his MS in mathematical engineering from Kyoto University in 1973. Mr. Ishigaki is a member of the Information Processing Society of Japan.

Kazuhiko Kushima is a Senior Research Engineer, Supervisor, Database Systems Laboratory NTT Information and Communication Systems Laboratories. His research interests include information retrieval on multimedia information. He received his BE in electronic engineering from Kyoto University in 1980.

Tetsuo Sakata is a Research Engineer, Database Systems Laboratory NTT Information and Communication Systems Laboratories. His research interests include information retrieval on multimedia information. He received his MS in applyed system science from Kyoto University in 1990. 